\title{
A MONITORING SYSTEM FOR PLC CONTROLLED MANUFACTURING SYSTEM BASED ON FIELDBUS
}

\author{
Xiaofeng Song ${ }^{1}$, Shili Tan ${ }^{2}$ and Junjian Ding ${ }^{3}$ \\ 'Department of Precise Machinery, Shanghai University, Shanghai, 200072. \\ Email:mail2song@163.com. 2Department of Precise Machinery, Shanghai University, \\ Shanghai. ${ }^{3}$ Department of Precise Machinery, Shanghai University, Shanghai.
}

Abstract: In this paper, we propose a monitoring system for Programmable Logic Controllers (PLCs) controlled manufacturing system based on CC-Link fieldbus in the process monitoring and control for film plating. The PLCs are mainly utilized for collecting data as well as realizing auto-tuning PID and sequence control strategies. In addition, Mitsubishi GT Designer software with a Human-Machine Interface (HMI) is used to monitor the dynamical process.

Key words: PLC; fieldbus; CC-Link; HMI; PID; monitoring system; winding system.

\section{INTRODUCTION}

The winding vacuum systems for plating metal such as zinc and aluminium on thin film are very common in the capacitance industry. The main goal is to increase as much as possible the film transport velocity while controlling the tension of the film. The tension and the speed of the film should be maintained at a reference value. The main concern is the coupling existing between film velocity and tension. There exist many sources of disturbances on the velocity (roller non-circularity, film sliding). If the delivery speed fluctuates, then the treatment on the film becomes nonuniform. On the other hand inappropriate tension may cause wrinkles or even a film break. Once film break takes place, the operator needs open the winding chamber again. Obviously the pressure of winding chamber comes back to standard atmosphere pressure. And it will take about 20 30 minutes for winding chamber to reach the required pressure (about $1.3 \times 10-1 \sim 2.67$

Please use the following format when citing this chapter:

Song, Xiaofeng, Tan, Shili, Ding, Junjian, 2006, in International Federation for Information Processing (IFIP), Volume 207, Knowledge Enterprise: Intelligent Strategies In Product Design, Manufacturing, and Management, eds. K. Wang, Kovacs G., Wozny M., Fang M., (Boston: Springer), pp. 576-581. 
$\times 10-2 \mathrm{~Pa}$ ). Accordingly the output will decrease evidently. So a monitoring system to monitor tension fluctuation in order to avoid film break is very needful.

CC-Link is a field network system that processes both control and information data at high speed, to provide efficient, integrated factory and process automation. Using CC-Link enables users to reduce the amount of control and power wiring required in the complicated production line. Since users can select the suitable devices from more than 354 field devices supported CC-Link it is easy to expand multi-vendor environment.

As for the communication of speed, this enables the communication with the sensor input which requires a high speed response and intelligent devices which requires a large amount of data communication.

As one of CC-Links features, RAS functions such as, stand-by master function, detaching slave station function and auto return function enable recovery from the communication fault and the system debug at start up time. As for test and monitoring function, confirmation of the data link status, hardware test and circuit test are available. The CC-Link network diagram is shown as Fig. 1. The CC-Link monitoring system is composed of master station 、slave stations 、 personal computer 、 inverter 、 servo motors 、 remote $\mathrm{I} / \mathrm{O}$ units, ect.

In order to meet the quest for automation and flexibility, many complex manufacturing systems are controlled by Programmable Logical Controllers (PLC). This is because that PLC's are adaptable, modular, user-friendly and acquired at low cost. And that winding vacuum film plating system is just such a complex manufacturing system.

The paper presents an intelligent monitoring system based on CC-Link fieldbus which consists of upper personal computer and the lower Programmable Logic Controllers for the aim of control and information management. A new kind of technology using CC-Link fieldbus is adopted in this monitoring system.

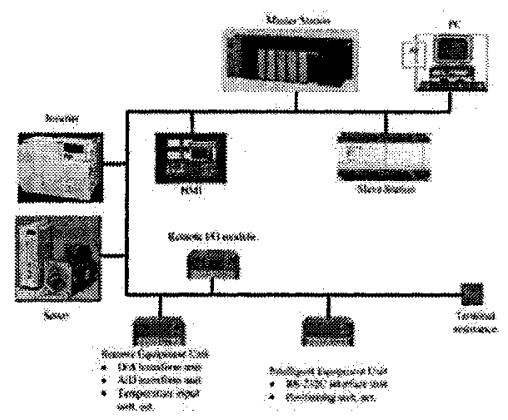

Figure 1. CC-Link network diagram 


\section{STRUCTURE OF THE MONITORING SYSTEM}

This fieldbus-based monitoring system has two arrangements including monitoring units in field and information management and control unit in control room. All intelligent monitoring units located nearby the winding vacuum machine have microprocessors, which have the following function as respective signal sampling, A/D conversion, data calculation, ect. Fieldbus is the most important connection between each discrete monitoring unit in slave stations and master station. Analog signal is superseded by digital signal for bi-directional communication. It is beneficial for an operator to realize control, verification and parameter setting in control room. The kind of structure, on one hand, increases the precision and antiinterference capability of the monitoring system. On the other hand, it saves the investment cost.

The quantities of intelligent monitoring units should be determined by practical needs. This system has five kinds of monitoring units: temperature, vacuum degree, roll diameter, film tension and transfer velocity.

Whether the monitoring system could work normally depends on the performance of the sensors to a considerable degree. Their precision and stability has a direct influence on the monitoring system. Data obtained from these sensors are processed by PLC and displayed on HMI as Fig. 2 shown.

Intelligent monitoring module has two functions: on one hand it filters, amplifies and adjusts the output signal got from sensors to appropriate value which can be processed by PLC (FX series). On the other hand it carries through signal sampling, A/D conversion, data processing, software antiinterference, parameter calculation and transmits data to central control unit by virtue of communication interface of a PLC.

Central control unit $(\mathrm{CCU})$ is the center of this monitoring system which consists of PC and RS232. It takes control of working condition of each intelligent monitoring unit, gets data and abnormal information and saves them. In addition, CCU can be visited by upper user computers. Furthermore, it has been practicable to analyze data and give a diagnosis for winding system by taking advantage of data management and fault diagnosis software.

The monitoring system makes use of CC-Link fieldbus which consists of communication module and twisted-pair wire acting as communication medium. Sixty-four stations could be connected to this kind of fieldbus at most. The communication module is connected with monitoring units and respective stations through CC-Link fieldbus. Transmission distance even reaches $1.2 \mathrm{~km}$ while communication rate of fieldbus is $156 \mathrm{kpbs}$. 


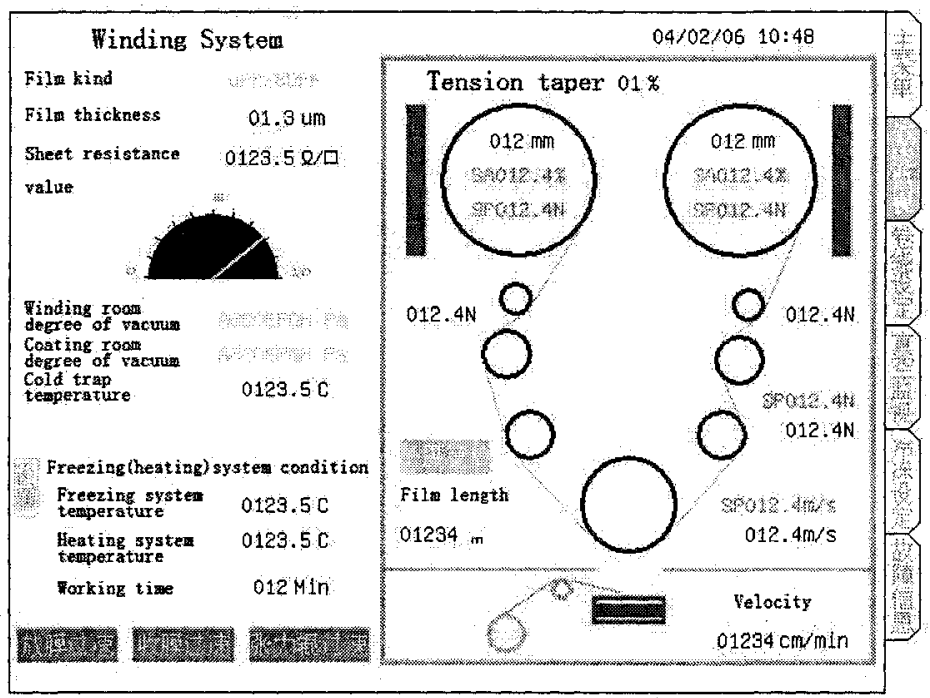

Figure 2. Monitoring display on HMI

\section{PLC PROGRAMMING}

In this winding system, we use Mitsubishi A series PLC as master station PLC because it has the characteristic of quick response and great ability of information processing. It is used to control the behaviors of the total winding system together with FX series PLCs of winding and unwinding system. The operating actions of the system and the sequence of these actions were edited beforehand into the control program by the designer. The control program sets a series of operations of the winding system, which tells the PLCs how to control a system. The current states of all sensors or actuators are saved as an array of input, output or flag signals in the PLC memory. Therefore, the PLC program is the basis of monitoring in a PLC controlled manufacturing system.

The programming method used is the ladder diagram method. The PLC system provides a design environment in the form of software tools running on a host computer terminal which allows ladder diagrams to be developed, verified, tested, and diagnosed. First, the high-level program is written in diagrams. Then, the ladder diagram is converted into binary instruction codes so that they can be stored in random-access memory (RAM) or erasable programmable read-only memory (EPROM). Each successive instruction is decoded and executed by the CPU. The function of the CPU is 
to control the operation of memory and $\mathrm{I} / \mathrm{O}$ devices and to process data according to the program. Each input and output connection point on a PLC has an address used to identify the I/O bit. The method for the direct representation of data associated with the inputs, outputs, and memory is based on the fact that the PLC memory is organized into three regions: input image memory, output image memory, and internal memory[4].

The PLC program uses a cyclic scan in the main program loop such that periodic checks are made to the input variables. The program loop starts by scanning the inputs to the system and storing their states in fixed memory locations (input image memory). The ladder program is then executed rungby-rung. Scanning the program and solving the logic of the various ladder rungs determine the output states. The updated output states are stored in fixed memory locations (output image memory). The output values held in memory are then used to set and reset the physical outputs of the PLC simultaneously at the end of the program scan[4].

As we know, logic control is a remarkable property of PLC, and it can be applied to efficiently handle analog data[2].

1) Analog data acquisition and transformation

Analog inputs and outputs such as pressure and temperature need to be measured on-line. For instance, temperature is first acquired by the Platinum resistances. A signal transformation module then converts the measured resistance value into the voltage range of $1-5 \mathrm{~V}$. The output of this transformation module is finally collected and transmitted back to the above PLC.

2) PLC control algorithms

The analog variables could be any controlled variable, e.g., temperature or pressure in our winding system. As a matter of fact, there exist two control modes, auto and manual. In the manual mode, operators modify the output values according to the desired levels; while in the auto mode, output values are regulated by the pre-designed control algorithms. It is worth mentioning that the outputs of PLC are always incremental values. Although auto-tuning PID controllers can be employed here, manual tuning is always used at the beginning of the production for routine initialization, and after that the system is switched to the auto mode. We emphasize that user's experiences oriented fuzzy logic controllers are utilized here to further improve the production performance as well. A film tension control system using fuzzy logic controller (FLC) on PLC to cope with the winding system uncertainty is proposed. Fuzzy logic algorithm is realized by basic PLC instructions.

To deal with the system with fixed model and parameter conventional PID is enough, but fixture is relative, in fact, the parameters of the winding system are variable in a definite range, therefore the controller must has 
robustness to obtain high performance. For this purpose, fuzzy reasoning is used to adjust the factors of PID, by this way the PID factors can be adjusted according to the changes of system state and plant parameters.

3) Servo motors control

At the beginning the film is transferred at a low speed, and then the servo motors are accelerated to set speed to wind at high speed at about 8 miles per second. The film tension is detected by load cells which control servo motors velocity to keep tension constant. The objective of control is not only to keep film tension constant but also to make winding velocity arrive at set value at least time. So the main problem is how to control servo motors accurately.

\section{CONCLUSION}

A monitoring system for PLC controlled manufacturing System Based on fieldbus for film plating production process is presented in this paper. It has been successfully on operation in practical production since 2003. Promising results have demonstrated its great advantage in practice as well as significant profit improvement.

\section{ACKNOWLEDGMENT}

The research has been supported by Shanghai Educational Committee and Shanghai Kelai mechatronics engineering Co., Ltd..

\section{REFERENCES}

1. J. C. Xing, P. Wang, B. Y. Cheng, S. Q. Wang, and Q. L. Yang, "Fieldbus Based Distributed Control System," 8th International Conference on Control, Automation, Robotics and Vision Kunming, China, 6-9th December 2004, pp. 1690-1694.

2. Q. Sheng, X. Z. Gao, and X. Y. Zhuang, "PLC-based Control Systems for Industrial Production of Fuel Alcohol" IEEE IClT' 02, Bangkok, THAILAND, pp. 827-832.

3. W. Hu, M. Schroeder, and A. G. Starr, "A Knowledge-Based Real-Time Diagnostic System for PLC Controlled Manufacturing Systems," 1999 IEEE, pp. 499-504.

4. Maria G. Ioannides, "Design and Implementation of PLC-Based Monitoring Control System for Induction Motor," IEEE Transactions On Energy Conversion, VOL.19, NO.3, September 2004, pp. 469-476.

5. Y. M. Jia, F. H. Su, J. Liu, “An On-line Insulation Monitoring System Based On Fieldbus”, pp. 769-772. 